\title{
Study on Plant Nutrition Indicator Using Leaf Spectral Transmittance for Nitrogen Detection
}

\author{
Juanxiu $\mathrm{Hu}^{1}$, Dongxian $\mathrm{He}^{1, *}$, and Po Yang ${ }^{2}$ \\ ${ }^{1}$ Key Lab. of Agriculture Engineering in Structure and Environment, \\ College of Water Conservation and Civil Engineering, China Agricultural University, \\ Beijing, P.R. China \\ he_dongxian@hotmail.com \\ ${ }^{2}$ Beijing Lighting valley Technology Company, Beijing, P.R. China
}

\begin{abstract}
The low fertilizer utilization at growing season and environment pollution coursed by unreasonable fertilization are becoming global outstanding problems in agricultural production. Scientific and reasonable fertilization based on rapid and nondestructive plant nutrient detection will be a valuable solution for solving above problems. In this study, spectral transmittance in wavelength ranged from 300 to $1100 \mathrm{~nm}$, chlorophyll content and nitrogen content of rice and cucumber leaves treated with culture solution in five different nitrogen levels were measured. According to the correlation analysis between them, 560, 650, and 720 $\mathrm{nm}$ as feature wavelengths and $940 \mathrm{~nm}$ as reference wavelength were determined for nitrogen detection. Correlation analysis between 21 spectral feature parameters composed by the transmittance at above wavelength, the leaf chlorophyll content and nitrogen content, and combined with their regression examination indicated that spectral feature parameters of (T940 - T560) / (T940 + T560), log (T940/T560) and $\log$ (T940/T650) are useful to conduct plant nutrient diagnosis with less than $8 \%$ relative error in rice and cucumber leaves. Therefore, the above spectral feature parameters as plant nitrogen indicators can be used to estimate the chlorophyll content and nitrogen content, furthermore support for non-destructive plant nutrient detection and fertilizer recommendation based on testing soil.
\end{abstract}

Keywords: Chlorophyll content, Nitrogen content, Spectral feature parameter, Spectral transmittance.

\section{Introduction}

Nitrogen is an essential nutrient factor for growth and quality of plants. The applying of nitrogen fertilizer has been growing persistently in order to increase the crop yield. However, the environmental pollutions caused by low seasonal utilization of nitrogen fertilizer and the unreasonable applications of chemical fertilizer have become outstanding issues in agricultural production $[1,2]$. The amount of nitrogen in soil is closely related to the nitrogen nutrition status of plant. The scientific nitrogen fertilization based on evaluating nitrogen nutrition status of plant is an effective approach to solve the problem above.

\footnotetext{
* Corresponding author.
} 
Chlorophyll content and nitrogen content are important indications for evaluating nitrogen nutrition status of plant. Estimating chlorophyll content and nitrogen content by methods of chemical analysis though presenting reliable results, cannot meet the requirement of guiding fertilizer promptly because of the vary complicated and time-consuming process [3]. The application of remote sensing technology in agriculture has provided a new means for rapid and non-destructive diagnosis of plant nitrogen nutrition [4]. There are lots of studies focusing on diagnosis of plant nitrogen nutrition based on the spectral properties of plant were carried out. Spectral reflectance of plant leaves in the vicinity of 550 and $670 \mathrm{~nm}$ were highly relevant to chlorophyll and nitrogen contents by measuring the reflectance of leaves or canopy [5, 6]. Reflectance in $550 \mathrm{~nm}$ and $710 \mathrm{~nm}$ could be used to better estimate the nitrogen content in corn and $R(550 \sim 600) / R(800 \sim 900)$ could be sensitive to reflect the nitrogen stress in corn. The linear combinations of reflectance in 620 and $760 \mathrm{~nm}$ had good correlation with nitrogen contents in rice leaves [7].

As to studies on plant spectral transmittance and nitrogen nutrition, an in-vivo detection device was developed for estimation plant chlorophyll content based on the difference in leaf spectral transmittance between in 660-690 nm and in 760-1100 nm [8]. Spectral characteristic parameter log (I660-690/I760-1100) was adopted in the device to estimate leaf chlorophyll content, in which I660-690 and I760 1100 were the intensity of light within corresponding wavebands having transmitted through leaves. Minolta, a Japanese company, then improved the algorithm and developed a portable chlorophyll meter. The spectral feature parameter adopted in the meter was log (T940/T650), namely, SPAD (Soil and Plant Analysis Development) value, in which T940 and T650 were the transmittance in corresponding wavelengths. The portable chlorophyll meter has been widely applied in rapid and non-destructive diagnosis of nitrogen nutrition in rice, maize, wheat, cotton and other field corps [9-12]. It also had a good performance in application for horticultural plants and xylophyta such as sweet potato, rape, peach, and maple, etc [13-16].

Many studies have been conducted on nitrogen nutrition diagnosis with the plant reflection spectrum, while the research on plant spectral transmittance is mostly based on the application of the portable chlorophyll meter. In this study, the feature band in direct relation with nitrogen nutrition of plants was determined based on transmittance spectra of rice and cucumber leaves cultivated in nutrient solution of different levels of nitrogen fertilizer. The correlation analysis and regression estimation error analysis were carried out of established spectral feature parameters and chlorophyll content as well as nitrogen contents, so as to determine plant nutrition indicators for nitrogen detection through estimating chlorophyll content and nitrogen content in leaves.

\section{Materials and Methods}

\subsection{Cultivation of Plants}

In this study, the cultivation of plants were performed in a environment controlled greenhouse using cucumber (Cucumis sativus L., cv., Zhongnong No.8 and Beijing 203) and rice (Oryza sativa L., cv., Wuyujing No. 3) as model plant during the period from 2008 to 2009. Cucumber and rice seedlings were transplanted to 5 cultivation 
beds of the same dimension $(250 \mathrm{~cm} \times 60 \mathrm{~cm} \times 40 \mathrm{~cm})$, and 16 plants in each bed. A homogenous mixture of vermiculite, grass peat and perlite at a proportion of 3:1:1 was used as the cultivation base. The temperature and light intensity in greenhouse were optimumly controlled through pad and fan cooling system and sunshade screen.
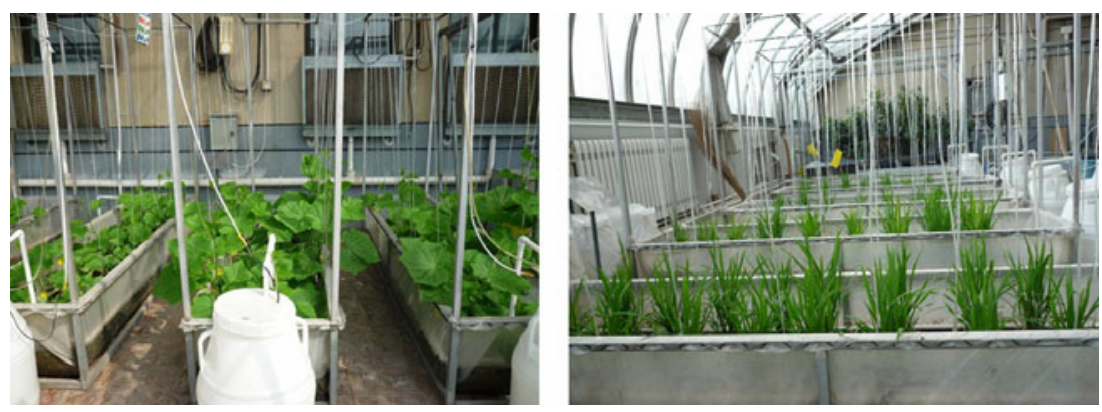

Fig. 1. The status of cucumber and rice cultivation

Cucumber and rice plants were treated with culture solution in 5 different nitrogen levels. With the nitrogen level of nutrient solution formulation for cucumbers from Yamazaki Japan as $100 \%$, content of nitrogen in the nutrient solutions were increased or decreased by $50 \%$ and $100 \%$ respectively while content equality of other major nutrient elements was ensured as far as possible (Table 1). Then the growing areas, according to the different nitrogen levels, were marked as N0, N50, N100, N150 and N200. The pH of culture solution was adjusted to 6.5-7.5. Pumps and timers were applied in automatic timing irrigation of the nutrient solution.

Table 1. Nutrient solution in different treatments for cucumber and rice cultivation

\begin{tabular}{lccccc}
\hline & \multicolumn{5}{c}{ The content of chemical in nutrient solutions (Unit: mg L $^{-1}$ ) } \\
\cline { 2 - 6 } & $\mathrm{N} 0$ & $\mathrm{~N} 50$ & $\mathrm{~N} 100$ & $\mathrm{~N} 150$ & $\mathrm{~N} 200$ \\
\hline $\mathrm{Ca}\left(\mathrm{NO}_{3}\right)_{2} \cdot 4 \mathrm{H}_{2} \mathrm{O}$ & 0 & 0 & 826 & 826 & 826 \\
$\mathrm{CaCl}_{2} \cdot 2 \mathrm{H}_{2} \mathrm{O}$ & 516 & 516 & 0 & 0 & 0 \\
$\mathrm{KNO}_{3}$ & 0 & 607 & 607 & 607 & 607 \\
$\mathrm{KCl}_{\mathrm{KH}}$ & 370 & 0 & 0 & 0 & 0 \\
$\mathrm{PO}_{4}$ & 136 & 0 & 0 & 0 & 0 \\
$\mathrm{NH}_{4} \mathrm{H}_{2} \mathrm{PO}_{4}$ & 0 & 115 & 115 & 115 & 115 \\
$\mathrm{NH}_{4} \mathrm{NO}_{3}$ & 0 & 0 & 0 & 280 & 560 \\
$\mathrm{MgSO}_{4} \cdot 7 \mathrm{H}_{2} \mathrm{O}$ & 483 & 483 & 483 & 483 & 483 \\
\hline & The content of major nutrient elements (Unit: $\left.\mathrm{mmol} \mathrm{L}^{-1}\right)$ \\
\hline $\mathrm{N}$ & $\mathrm{N} 0$ & $\mathrm{~N} 50$ & $\mathrm{~N} 100$ & $\mathrm{~N} 150$ & $\mathrm{~N} 200$ \\
\hline $\mathrm{P}$ & 0 & 7 & 14 & 21 & 28 \\
$\mathrm{~K}$ & 1 & 1 & 1 & 1 & 1 \\
$\mathrm{Ca}$ & 6 & 6 & 6 & 6 & 6 \\
$\mathrm{Mg}$ & 3.5 & 3.5 & 3.5 & 3.5 & 3.5 \\
\hline
\end{tabular}




\subsection{Measured Parameters and Measurement Methods}

During the tillering stage of rice and fast growth period of cucumber, five samples of plant leaves in each treatment area were selected respectively for parameters measurement of the spectral property and nitrogen nutrition indices, including the spectral transmittance of leaf within the wavelength range of $300-1100 \mathrm{~nm}$, and the chlorophyll content and nitrogen content of leaf.

(1) The determination of spectral transmittance

Spectral transmittance of rice or cucumber leaves was determined using an integrating sphere measurement device in the large sample chamber of spectrophotometer (UV3150, Shimadzu Co., Japan). The measurement wavelength range was 300-1100 $\mathrm{nm}$ with the interval of $1 \mathrm{~nm}$. The light source switch point was set at $360 \mathrm{~nm}$ and the raster switch point at $820 \mathrm{~nm}$ while the slit width was $20 \mathrm{~nm}$. The measurement position was basically in both sides of the main vein and middle of the leaf. For each leaf, 4 different positions were selected for determination of spectral transmittance, so each leaf was measured for 4 times.

(2) The determination of chlorophyll content

$0.05 \mathrm{~g}$ rice leaves $(0.20 \mathrm{~g}$ cucumber leaves) were weighed, cut into filaments and put into test tubes. After adding 10mL mixture of 1:1 ethanol-acetone, the tubes were placed in refrigerator at $4^{\circ} \mathrm{C}$ for $16-18 \mathrm{~h}$ in the dark for extracting chlorophyll of leaves. The extract was uniformly shaken and then transferred into a clean cell for determination of chlorophyll content. With the ethanol-acetone (1:1) mixture as the reference, absorbance values at 663 and $645 \mathrm{~nm}$ of the extract were measured by a spectrometer (UV3150, Shimadzu Co., Japan).The chlorophyll content was calculated by the correction formula of Arnon method [17].

(3) The determination of nitrogen content

$0.10 \mathrm{~g}$ rice or cucumber leaves were weighed to a $100 \mathrm{~mL}$ digestion tubes and wetted with a little water dripped in, and then $4 \mathrm{~mL}$ of concentrated $\mathrm{H}_{2} \mathrm{SO}_{4}$ was added. The tubes were covered with small curved neck funnels to place overnight after shaken gently. The next day, the tubes were put into a digestion furnace to heat firstly at $180^{\circ} \mathrm{C}$ for an hour, and then at $300^{\circ} \mathrm{C}$ for 2 hours. When the solution became brown-red, the tubes were moved away and cooled for $3 \mathrm{~min}$. Then 15 drops of $30 \% \mathrm{H}_{2} \mathrm{O}_{2}$ were added into the solution. The tube was put into the digestion furnace to heat at $300^{\circ} \mathrm{C}$ again for 15 min after shaken. Then 10 drops of $30 \% \mathrm{H}_{2} \mathrm{O}_{2}$ was added after the tubes were moved away to cool for $3 \mathrm{~min}$ again. The above procedures were repeated for times with the amount of $30 \% \mathrm{H}_{2} \mathrm{O}_{2}$ gradually decreased until the solution became colorless or bright. Finally, the tubes were heated for 20 min in order to remove residual $\mathrm{H}_{2} \mathrm{O}_{2}$. After thoroughly cooled, the digestion solution was arranged on a Kjeldahl type automatic azotometer (KDY-9830, Beijing Tongrunyuan Electromechanical Technology Co., Ltd., China) for distillation titration to determine the nitrogen content.

\subsection{Processing and Analysis of Data}

The mean value of four measurements of each leaf sample's spectral transmittance within 300-1100 nm was adopted. The correlation analysis of the leaf spectral 
transmittance, the chlorophyll content, and the nitrogen content was conducted with the SPSS software, so as to determine the feature wavelength and reference wavelength for nitrogen nutrition diagnosis in plants. With the correlation analysis of spectral feature parameters established by the spectral transmittance of characteristic wavelength and reference wavelength, the leaf chlorophyll content, and the leaf nitrogen contents, plant nitrogen nutrition indicators were determined and tested.

\section{Results and Analysis}

\subsection{The Spectral Transmission of Plant Leaves}

Spectral transmittances of cucumber and rice leaves were changed basically with the same trend of the wavelengths in different nitrogen nutrition levels. In the range of photosynthetically active radiation wave band $(400-700 \mathrm{~nm})$, the leaf spectral transmittance formed wave crest at $550 \mathrm{~nm}$ and shaped up wave trough at $680 \mathrm{~nm}$. At the same time, the leaf spectral transmittance reduced with the increasing nitrogen. Spectral transmittances of leaves were all between 40 50\% in near-infrared wave band (800-1100 $\mathrm{nm})$, in which the range of variation was little.
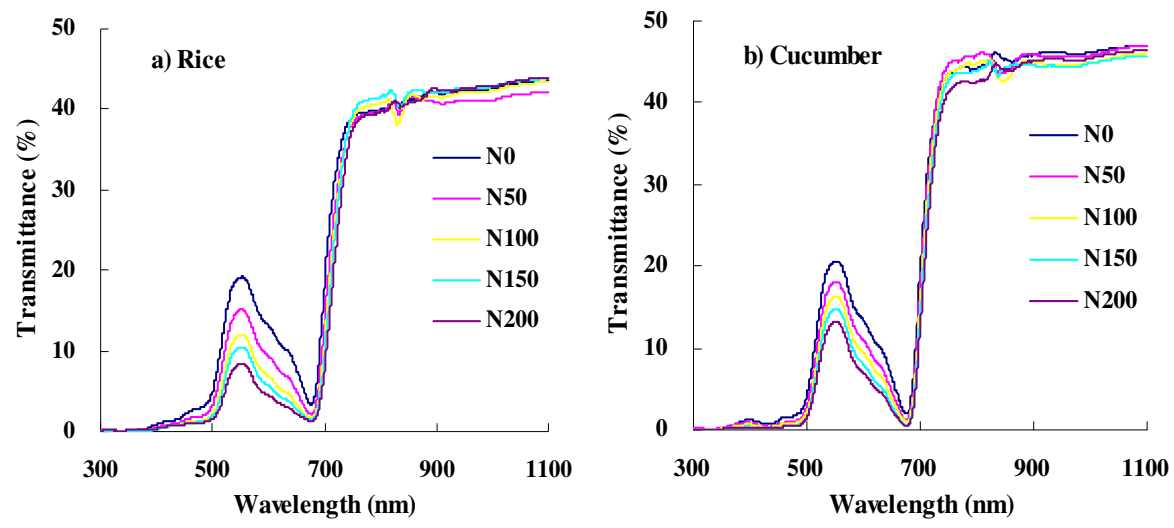

Fig. 2. Leaf spectral transmittances of cucumber and rice leaves treated with culture solution in different nitrogen levels

The spectral properties of plant leaves are mainly formed by absorption, reflection and transmission of light towards mesophyll cells, chlorophyll, moisture content and other biochemical components in the leaves. In the wave band of $400-700 \mathrm{~nm}$, the spectral properties of leaves were mainly affected by chlorophyll. Due to strong absorption of red light and little absorption of green light by leaf chlorophyll, a wave crest was formed around $550 \mathrm{~nm}$ while a wave trough of low transmittances around 680 $\mathrm{nm}$ in leaf spectral transmittance. In fast growth period of plants, with the increasing of fertilizer nitrogen, the chlorophyll and nitrogen content also increased so that leaves could absorb more light at $400-700 \mathrm{~nm}$ for photosynthesis, therefore spectral transmittance of plant leaves in this wave band decreased with the increasing of 
fertilizer nitrogen. In the near-infrared wave band $(800-1100 \mathrm{~nm})$, the spectral properties of leaves were mainly influenced by the tissue structure in leaves, while the impact of chlorophyll could be negligible. The spectral transmittance of leaf in the near-infrared wave band was $40-50 \%$, which is in line with the results of plant leaf spectrum by other researchers referring to that the absorptivity of leaf was generally less than $10 \%$ and reflectivity was about $50 \%$ [18-19].

\subsection{The Correlation between Spectral Transmittance of Leaves and Chlorophyll Contents as well as Nitrogen Contents}

The significant negative correlation between spectral transmittance in the waveband of 500-720 nm and leaf chlorophyll content as well as nitrogen content was found in rice and cucumber leaves with the correlation coefficients ranging from -0.8 to -0.9 $(\mathrm{P}=0.01)$. Within the waveband of $900-1100 \mathrm{~nm}$, there was not a significant correlation between spectral transmittance and chlorophyll content as well as nitrogen content.
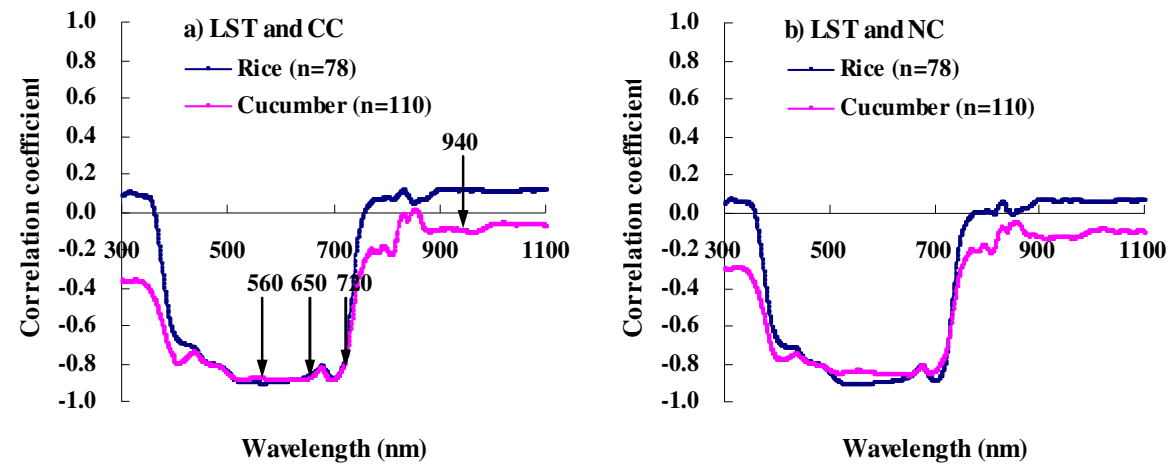

Fig. 3. Correlation coefficient between leaf spectral transmittance (LST), chlorophyll content (CC) and nitrogen content (NC)

Integrated spectral feature parameters, such as difference value and ratio of spectral parameters between feature waveband and reference waveband were adopted in order to minimize the impact of structure and thickness of plant leaves on the spectral data analysis thereby enhancing the accuracy of estimations of chlorophyll and nitrogen content[6, 7]. For instance, spectral feature parameter of log (T940/T650) was used in the portable chlorophyll meter (SPAD-502) produced by Japanese company Minolta to estimate leaf chlorophyll content. A portable meter developed by Fuchigami et al for plant nutrient detection adopts T940/T560 and T940/T720 [20]. Based on the correlation between spectral transmittance and chlorophyll content as well as nitrogen content and relevant studies, 560, 650, $720 \mathrm{~nm}$ were selected as typical feature wavelengths in the 500-720 nm waveband, and at the same time $940 \mathrm{~nm}$ was chosen within the wave band of $900-1100 \mathrm{~nm}$ as the reference wavelength to establish 21 spectral feature parameters (Table 2) for nitrogen nutrition diagnosis of plants. 


\subsection{The Correlation between Spectral Feature Parameters of Leaves and Chlorophyll Contents as well as Nitrogen Contents}

The spectral feature parameters the correlation coefficient between which and chlorophyll content in rice was over $0.94(\mathrm{P}=0.01)$ were as follows: T940/T560, (T940 - T560) / (T940 + T560), (1/T560 - 1/T940) / (1/T940), (T940 - T560) / T940, $\log$ (T940/T560), T940/T650, (1/T650 - 1/T940) / (1/T940), log (T940/T650). Moreover, the correlation coefficient between all spectral feature parameters mentioned above and nitrogen contents in rice was over $0.91(\mathrm{P}=0.01)$ (Table 2). The spectral feature parameters the correlation coefficient between which and chlorophyll content in cucumber was over $0.91(\mathrm{P}=0.01)$ were as follows: (T940 - T560) / (T940 + T560), log (T940/T560), (T940 - T650) / (T940 + T650), log (T940/T650). The correlation coefficient between the two former spectral feature parameters and nitrogen content in cucumber was 0.87 and 0.88 respectively, while that of the latter two and nitrogen content was over $0.90(\mathrm{P}=0.01)$. It was indicated that the spectral feature parameters of (T940 - T560) / (T940 + T560), log (T940/T560), and log (T940/T650) had a high correlation with chlorophyll and nitrogen contents in cucumber and rice.

Table 2. Correlation coefficient between spectral feature parameters and chlorophyll content as well as nitrogen content

\begin{tabular}{|c|c|c|c|c|}
\hline & \multicolumn{2}{|c|}{$\begin{array}{l}\text { spectral feature parameter } \\
\text { and chlorophyll content }\end{array}$} & \multicolumn{2}{|c|}{$\begin{array}{l}\text { spectral feature parameter } \\
\text { and nitrogen content }\end{array}$} \\
\hline & rice & cucumber & rice & cucumber \\
\hline T940/T560 & 0.94 & 0.90 & 0.93 & 0.87 \\
\hline$(\mathrm{T} 940-\mathrm{T} 560) /(\mathrm{T} 940+\mathrm{T} 560)$ & 0.95 & 0.91 & 0.92 & 0.87 \\
\hline (T940 - T560) / T940 & 0.94 & 0.90 & 0.91 & 0.87 \\
\hline T940 - T560 - T940 × T560 & 0.92 & 0.89 & 0.88 & 0.86 \\
\hline$(1 / \mathrm{T} 560-1 / \mathrm{T} 940) /(1 / \mathrm{T} 940)$ & 0.94 & 0.90 & 0.93 & 0.87 \\
\hline $\log (\mathrm{T} 940 / \mathrm{T} 560)$ & 0.95 & 0.91 & 0.93 & 0.88 \\
\hline $\log (\mathrm{T} 940 / \mathrm{T} 560) / \log (\mathrm{T} 940)$ & -0.92 & -0.88 & -0.87 & -0.84 \\
\hline T940/T650 & 0.94 & 0.89 & 0.92 & 0.87 \\
\hline$(\mathrm{T} 940-\mathrm{T} 650) /(\mathrm{T} 940+\mathrm{T} 650)$ & 0.91 & 0.91 & 0.89 & 0.90 \\
\hline (T940 - T650) / T940 & 0.90 & 0.90 & 0.88 & 0.89 \\
\hline T940 - T650 - T940 × T650 & 0.76 & 0.78 & 0.71 & 0.76 \\
\hline$(1 / \mathrm{T} 650-1 / \mathrm{T} 940) /(1 / \mathrm{T} 940)$ & 0.94 & 0.89 & 0.92 & 0.87 \\
\hline $\log (\mathrm{T} 940 / \mathrm{T} 650)$ & 0.94 & 0.92 & 0.92 & 0.90 \\
\hline $\log (\mathrm{T} 940 / \mathrm{T} 650) / \log (\mathrm{T} 940)$ & -0.88 & -0.89 & -0.84 & -0.88 \\
\hline $\mathrm{T} 940 / \mathrm{T} 720$ & 0.90 & 0.86 & 0.88 & 0.81 \\
\hline$(\mathrm{T} 940-\mathrm{T} 720) /(\mathrm{T} 940+\mathrm{T} 720)$ & 0.90 & 0.86 & 0.88 & 0.81 \\
\hline (T940 - T720) / T940 & 0.90 & 0.86 & 0.88 & 0.80 \\
\hline T940 - T720 - T940 × T720 & 0.89 & 0.85 & 0.87 & 0.80 \\
\hline$(1 / \mathrm{T} 720-1 / \mathrm{T} 940) /(1 / \mathrm{T} 940)$ & 0.90 & 0.86 & 0.88 & 0.80 \\
\hline $\log (\mathrm{T} 940 / \mathrm{T} 720)$ & 0.90 & 0.86 & 0.89 & 0.81 \\
\hline $\log (\mathrm{T} 940 / \mathrm{T} 720) / \log (\mathrm{T} 940)$ & -0.92 & -0.84 & -0.88 & -0.78 \\
\hline
\end{tabular}




\subsection{The Experimental Examination of Spectral Feature Parameters}

Linear regression analysis was carried out of spectral feature parameters, namely (T940 - T560) / (T940 + T560), log (T940/T560) and log (T940/T650), and chlorophyll as well as nitrogen contents in rice and cucumber measured by chemical methods. Chlorophyll and nitrogen contents were estimated through the regression formula and compared with the values measured practically (Table 3). It was found that the relative errors of chlorophyll and nitrogen contents in rice estimated by (T940 - T560) / (T940 + T560), $\log$ (T940/T560), and $\log$ (T940/T650) were below 5.4\%. As regards to chlorophyll contents in cucumber, the relative errors were below $4.5 \%$, and the relative error of its nitrogen content was less than $7.7 \%$. These results indicated that the relative errors of estimated leaf chlorophyll and nitrogen contents with spectral feature parameters of (T940 - T560) / (T940 + T560), log (T940/T560), and log (T940/T650), could meet the requirements of testing the plant nitrogen nutrition in vivo. Thus, they are suitable for use indicators of plant nitrogen nutrition. Among the spectral feature parameters mentioned above, (T940 - T560) / (T940 + T560) was the same as was adopted in the plant nutrition detector developed by Fuchigami et al (2007) while log (T940/T650) was the same as used in the portable chlorophyll meter (SPAD-502) produced by Minolta Corporation.

Table 3. Relative error of estimated chlorophyll and nitrogen content using spectral feature parameter $(n=30)$

\begin{tabular}{lccccc}
\hline & \multicolumn{2}{c}{$\begin{array}{c}\text { Relative error of estimated } \\
\text { chlorophyll content }(\%)\end{array}$} & & \multicolumn{2}{c}{$\begin{array}{c}\text { Relative error of estimated } \\
\text { nitrogen content }(\%)\end{array}$} \\
\cline { 2 - 3 } \cline { 5 - 6 } \cline { 5 - 6 } & Rice & Cucumber & & Rice & Cucumber \\
\hline (T940- T560)/(T940+ T560) & $5.3 \pm 3.7$ & $4.4 \pm 3.5$ & & $5.2 \pm 3.4$ & $7.6 \pm 5.0$ \\
$\log (\mathrm{T} 940 / \mathrm{T} 560)$ & $5.2 \pm 3.7$ & $4.5 \pm 3.5$ & & $4.7 \pm 3.0$ & $7.7 \pm 5.0$ \\
$\log (\mathrm{T} 940 / \mathrm{T} 650)$ & $5.1 \pm 3.7$ & $4.5 \pm 3.9$ & & $5.4 \pm 3.4$ & $6.5 \pm 4.6$ \\
\hline
\end{tabular}

\section{Conclusions}

Spectral transmittance in wavelength ranged from 300 to $1100 \mathrm{~nm}$, chlorophyll content and nitrogen content of rice and cucumber leaves treated with culture solution in five different nitrogen levels were measured. According to the correlation analysis between them, 560, 650, and $720 \mathrm{~nm}$ as feature wavelengths and $940 \mathrm{~nm}$ as reference wavelength were determined for nitrogen detection. Correlation analysis between 21 spectral feature parameters composed by the transmittance at above wavelength, the leaf chlorophyll content and leaf nitrogen content, and combined with their regression examination indicated that spectral feature parameters of (T940 - T560) / (T940 + T560), $\log (\mathrm{T} 940 / \mathrm{T} 560)$ and $\log$ (T940/T650) are useful to conduct the plant nutrient diagnosis with less than $8 \%$ relative error in rice and cucumber leaves. Therefore, the above spectral feature parameters as plant nitrogen indicators can be used to estimate the chlorophyll content and nitrogen content. 
Acknowledgments. This work has been supported by National High Technology Research and Development Program of China (2006AA10A302) and (2006AA10Z206).

\section{References}

1. Zeng, X.B., Li, J.M.: Fertilizer Application and its Effect on Grain Production in Different Counties of China (in Chinese). Scientia Agricultura Sinica 37, 387-392 (2004)

2. Ju, X.T., Xing, G.X., Chen, X.P., Zhang, S.L., Zhang, L.J., Liu, X.J., Cui, Z.L.: Reducing Environmental Risk by Improving N Management in Intensive Chinese Agricultural Systems. Proceedings of the National Academy of Science 106, 3041-3046 (2009)

3. Jiao, W.J., Min, Q.W., Lin, K., Zhu, Q.K., Zhang, J.J.: Progress and Perspective on Nutrition Diagnosis of Plant Nitrogen (in Chinese). Chinese Agricultural Science Bulletin 22, 351-355 (2006)

4. Tian, Y.C., Zhu, Y., Yao, X., Liu, X.J., Cao, W.X.: Non-destructive Monitoring of Crop Nitrogen Nutrition Based on Spectral Information (in Chinese). Chinese Journal of Ecology 26, 1454-1463 (2007)

5. Thomas, J.R., Oerther, G.F.: Estimating Nitrogen Content of Sweet Pepper Leaves by Reflectance Measurements. Agronomy Journal 64, 11-13 (1972)

6. Filella, L., Serra, L., Penuelas, J.: Evaluating Wheat Nitrogen Status with Canopy Reflectance Indices and Discriminant Analysis. Crop Science 35, 1400-1405 (1995)

7. Shibayama, M., Akiyama, T.: A Spectroradiometer for Field Use VII. Radiometric Estimation of Nitrogen Levels in Field Rice Canopy. Japanese Journal of Crop Science 55, 433-438 (1986)

8. Watanabe, S., Kuzunuki, T.: Method of and Device for Measuring Chlorophyll of Living Leaves. Patent, Japan (1981)

9. Turner, F.T., Jund, M.F.: Chlorophyll Meter to Predict Nitrogen Topdress Requirement for Semidwarf Rice. Agronomy Journal 83, 926-928 (1991)

10. Wu, L.H., Tao, Q.N.: Nitrogen Fertilizer Application Based on the Diagnosis of Nitrogen Nutrition of Rice Plants (Oryza sativa L.) Using Chlorophyll Meter (in Chinese). Journal of Zhejiang Agricultural University 25, 135-138 (1999)

11. Piekielek, W.P., Fox, R.H.: Use of a Chlorophyll Meter to Predict Side-dress Nitrogen Requirements for Maize. Agronomy Journal 84, 59-65 (1992)

12. Follett, R.H., Follett, R.F.: Use of a Chlorophyll Meter to Evaluate the Nitrogen Status of Dryland Winter Wheat. Communications in Soil Science and Plant Analysis 23, 687-697 (1992)

13. Shaahan, M.M., EI-Sayed, A.A., Abou EI-Nour, E.A.A.: Predicting Nitrogen, Magnesium and Iron Nutritional Status in some Perennial Crops Using a Portable Chlorophyll Meter. Scientia Horticulturae 82, 339-348 (1999)

14. Chang, S.X., Robison, D.J.: Nondestructive and Rapid Estimation of Hardwood Foliar Nitrogen Status Using the SPAD-502 Chlorophyll Meter. Forest Ecology and Management 181, 331-338 (2003)

15. Li, H.Y., Ren, Q.P., Sun, S., Ma, H.Y.: Study on Relation between SPAD Value and Chlorophyll Contents in 10 Kinds Horticulture Woody Plants (in Chinese). Forestry Science and Technology 34, 68-70 (2009)

16. Su, Y.S., Guo, H.C., Yang, X.L.: Study on Correlations between SPAD Readings and Chlorophyll Content in Leaves of Sweet Potato, Dioscorea and Konjaku (in Chinese). Southwest China Journal of Agricultural Sciencea 22, 64-66 (2009) 
17. Lichtenthaler, H.K., Wellbuen, A.R.: Determinations of Total Carotenoids and Chlorophyll a and b Leaf Extracts in Different Solvents. Biochemical Society Transactions 11, 591-592 (1983)

18. Schepers, J.S., Blackmer, T.M., Wilhelm, W., Resende, M.: Transmittance and Reflectance Measurements of Corn leaves from Plants with Different Nitrogen and Water Supply. Journal of Plant Physiology 148, 523-529 (1996)

19. Bauerle, W.L., Weston, D.J., Bowdena, J.D., Dudley, J.B., Toler, J.E.: Leaf Absorptance of Photosynthetically Active Radiation in Relation to Chlorophyll Meter Estimates Among Woody Plant Species. Scientia Horticulturae 101, 169-178 (2004)

20. Fuchigami, L.H., Ding, P.H., Barnes, G.E.: Portable Meter to Measure Chlorophyll, Nitrogen and Water and Methods. Patent, USA (2007) 